CLINICAL STUDY

\title{
MIB-1 labeling index correlated with magnetic resonance imaging detected tumor volume doubling time in pituitary adenoma
}

\author{
Chih-Yi Hsu ${ }^{1,3}$, Wan-Yuo Guo ${ }^{2,4}$, Chen-Ping Chien ${ }^{2}$ and Donald Ming-Tak Ho ${ }^{1,3}$ \\ Departments of ${ }^{1}$ Pathology and Laboratory Medicine and ${ }^{2}$ Radiology, Taipei Veterans General Hospital, 201 Shih-Pai Road, Section 2, Taipei 11217, \\ Taiwan, ROC and Departments of ${ }^{3}$ Pathology and ${ }^{4}$ Radiology, National Yang-Ming University School of Medicine, Taipei 11221, Taiwan, ROC \\ (Correspondence should be addressed to D M-T Ho at Department of Pathology and Laboratory Medicine, Taipei Veterans General Hospital; \\ Email: mtho@vghtpe.gov.tw)
}

\begin{abstract}
Objective: The purpose of this study was to evaluate whether MIB-1 labeling index (LI) could be used to predict growth velocity of residual pituitary adenomas after surgery.

Materials and methods: One hundred and sixty pituitary adenomas which had not received other treatment modality except for surgery were collected. Each of them had at least two post-operative magnetic resonance imaging (MRI) studies with an interval of at least 1 month apart. Tumor volume doubling time (TVDT) was calculated for those in which volume increased.

Results: Post-operative tumor progression was noted in 54 (33.8\%) cases, of which 39 (72.2\%) cases were non-functioning adenomas. The MIB-1 LIs of the functioning and non-functioning adenomas were not significantly different. The median TVDT of these 54 cases was 34.6 months. The mean and median MIB-1 LI were 2.7 and 1.1 respectively (range 0.4-20.6). The MIB-1 LI was significantly correlated with $\log _{2}$ (TVDT) $(r=-0.363, P=0.007)$; when LI was $<0.8$, the TVDT of $90.5 \%$ cases was $\geq 2$ years.

Conclusions: Only one-third of the pituitary adenomas progressed after surgery, and their MIB-1 LIs were generally low. The MIB-1 LI was significantly correlated with the MRI detected TVDT of post-operative residual pituitary adenomas.
\end{abstract}

European Journal of Endocrinology 162 1027-1033

\section{Introduction}

Pituitary adenomas are usually treated by surgery. Conventional radiotherapy (RT) or gamma-knife radiosurgery (GKRS) could be used for primary treatment or post-operative residual tumors. Since radiotherapy is not without adverse effects, such as radionecrosis or radiation-induced tumors (1-3), the timing for using RT/GKRS to treat post-operative residual pituitary adenomas could be adjusted based on the growth rate of tumors.

The MIB-1 MAB recognizes Ki-67 protein, which is expressed over the entire cell cycle except for $G_{0}$ phase. It has been employed as an operational marker of cell proliferation for various types of tumors $(4,5)$. Many researchers have studied the MIB-1 labeling index (LI) of pituitary adenomas (6-27), and most of them were focused on its relationship with invasiveness or recurrence of the tumor. However, there has been no consensus on the above subjects. The comparisons of the results of different series were difficult since the inclusion criteria of most studies were different.
In this study, we intended to evaluate whether MIB-1 LI correlates with growth velocity of pituitary adenomas, which was through the evaluation of tumor volume doubling time (TVDT) calculated from serial magnetic resonance imaging (MRI) studies. There were four studies in the literature evaluating the relationship between MIB-1 LI and pituitary adenomas' growth rate or TVDT derived from serial image studies $(7,19,20$, 27). Three of the studies showed a significant inverse correlation between MIB-1 LI and growth rate of pituitary adenomas. However, all these studies only evaluated non-functioning tumors, and two of them also used data from mixed image studies, i.e. computerized tomography (CT) and MRI. The TVDT estimated in two studies was also different, one being $930 \pm 180$ days (mean \pm s.D.; range 200-2550 days) (7) and the other being $1836 \pm 3445$ days (506-5378 days) (20). The growth velocity was described as linear growth in one study (19), while it was concluded as exponentially linear growth in the other study (20).

In this study, we included both functioning and non-functioning pituitary adenomas, and used only MRI to calculate tumor volume and TVDT. 


\section{Materials and methods}

From January 1993 to April 2004, there were 569 cases (637 tumors) of pituitary adenomas in the surgical pathology file of the Taipei Veterans General Hospital, Taipei, Taiwan. Cases that had two or more MRI studies with an interval of more than 1 month apart and did not receive RT, GKRS, or medication such as bromocriptine during the entire course and for which the surgical intervention was not between the two MRI studies were included. Because only a few cases had two or more preoperative MRI studies, this study only included 160 cases that had two post-operative MRI studies.

All MRI examinations were conducted using 1.5 Tesla MR scanners. The MR scanning parameters might have changed over the 11-year period with the concerns of imaging system optimization. Nevertheless, T1-weighted coronal views of pituitary fossa with same scanning positioning in pre- and post-gadolinium-based contrast medium administration with 3-mm slice thickness/0-mm gap were included in all MRI examinations. Tumor delineation was performed retrospectively by an experienced neuroradiologist (W Y G) in a short studying time. The tumor volume, based on meticulous comparison of pre- and post-contrast coronal view MRI, was defined as the sum of all tumor slabs measured on each tumor slice multiplied by $3 \mathrm{~mm}$. For tumors that were not discernible from the first MRI, their volume was assigned to be $1 \mathrm{~mm}^{3}(0.001 \mathrm{ml})$.

The tumor volume of the second MRI $\left(V_{2}\right)$ was compared with that of the first MRI $\left(V_{1}\right)$. The TVDT was calculated in cases that increased in size during the observation period by the following formula: TVDT $=$ interval $\times \log _{2}(2) / \log _{2}\left(V_{2} / V_{1}\right)(28)$.

Paraffin-embedded tissue blocks of the study cases were recut for hematoxylin and eosin staining and immunohistochemical staining for Ki-67 (clone MIB-1, monoclonal, 1:75, Dako, Glostrup, Denmark; Dako ChemMate Detection Kit, peroxidase (ChemMate); microwaved three times for 5 min each time). Positive and negative controls were included with each batch of the sections to confirm the consistency of the analysis. Sections from one glioblastoma which had a known average MIB-1 LI of 43.3 (s.D. 2.1) were used as a positive control. The batch of MIB-1 staining was considered acceptable when the LI of the control section was within the range of 39-47.6. Normal brain tissues from autopsy were used as a negative control.

For the MIB-1 stainings, any distinct nuclear staining was recorded as positive. The MIB-1 LI was defined as the percentage of positive staining tumor cells. Five fields (more than 1000 tumor cells) from the area of maximal labeling were chosen for photograph at a magnification of $400 \times$. Computer-assisted manual counting using Image-Pro Lite (Media Cybernetics, L.P., Bethesda, MD, USA) was performed. Vascular components and hematogenous cells were excluded from the analysis.
The general data including date of birth, gender, presence or absence of hormonal and/or neurological symptoms, date of surgery, history of RT/GKRS, and medication were reviewed from the medical records. The adenoma types were obtained from the pathology report, which were classified based on immunohistochemical staining using MABs against prolactin, $\mathrm{GH}$, ACTH, $\beta$ FSH, $\beta \mathrm{LH}, \mathrm{TSH}$, and $\alpha$-subunit of pituitary hormones. Plurihormonal adenomas are tumors capable of producing two or more hormones that differ in chemical composition and immunoreactivity. Lactotroph adenoma, somatotroph adenoma, corticotroph adenoma, thyrotroph adenoma, and plurihormonal adenoma were further separated into functioning and non-functioning (silent) groups based on the presence or absence of hormonal symptoms, while gonadotroph adenoma and null cell adenoma were classified in the non-functioning group.

The significance of age, tumor volume, TVDT, and MIB-1 LI was tested by the Mann-Whitney test or Kruskal-Wallis test. The significance of gender and adenoma type was determined using the $\chi^{2}$ test or Fisher's exact test. The correlation between TVDT and MIB-1 LI was tested by Spearman's rank correlation coefficient and Pearson's product moment correlation coefficient. A linear regression model was used to generate an expression for MIB-1 LI when predicting TVDT. The optimal cut-off point of MIB-1 LI was using the point on the receiver operating characteristic (ROC) curve closest to $(0,1)$. The statistical difference was considered significant when the $P$ value was $<0.05$.

The materials used in this study consisted only of archival specimens from this department. The procedures followed ethical standards of the Declaration of Helsinki 1975, as revised in 1983 and approved by the Institutional Review Board of our institute.

\section{Results}

The general data of the 160 pituitary adenomas that had received only surgical treatment during the entire course and had two or more post-operative MRI studies are shown in Table 1. Post-operative progression was noted in $54(33.75 \%)$ cases, while $106(66.25 \%)$ cases were either stable or regress.

Of the 54 cases with post-operative tumor progression, their median age was 48.5 years (mean \pm s.D., $49.4 \pm 15.8)$. The median age of patients with functioning adenoma (36.0 years) was significantly less than that of those with non-functioning adenoma (53.0 years; $P=0.004$ ). The male-to-female ratio was 1.1:1.0. Female predominance was seen in patients with functioning adenomas. The study group included all types of adenomas except for corticotroph adenoma and thyrotroph adenoma.

Of the parameters evaluated between the progressive group and the non-progressive group, none of them had 
Table 1 General data of 160 cases of pituitary adenomas which had two post-operative MRI studies of at least 1 month apart and did not receive any treatments other than surgery prior to case selection. Data represented as $n(\%)$ or median (range).

\begin{tabular}{lccc}
\hline & Total & Progressive adenoma & Non-progressive adenoma \\
\hline Case number & $160(100)$ & $54(33.8)$ & $106(66.3)$ \\
Age & $46.0(14-83)$ & $48.5(20-73)$ & $45.0(14-83)$ \\
Gender & & & \\
$\quad$ Male & $84(100)$ & $28(33.3)$ & $56(66.7)$ \\
Female & $76(100)$ & $26(34.2)$ & $50(65.8)$ \\
Adenoma type & & & \\
PRL & $24(100)$ & $8(33.3)$ & $16(66.7)$ \\
GH & $3(100)$ & $1(33.3)$ & $2(66.7)$ \\
ACTH & $5(100)$ & $0(0)$ & $5(100)$ \\
Gn & $65(100)$ & $23(35.4)$ & $42(64.6)$ \\
Pluri & $47(100)$ & $17(36.2)$ & $11(68.8)$ \\
Null & $16(100)$ & $5(31.3)$ & \\
Functional status & & & $26(63.4)$ \\
Functioning & $41(100)$ & $15(36.6)$ & $80(67.2)$ \\
Non-functioning & $119(100)$ & $39(32.8)$ & $2.9(0.1-55.4)$ \\
Tumor volume (ml) & $2.7(0.001-55.4)$ & $2.6(0.001-44.4)$ & $0.9(0.1-7.7)$ \\
MIB-1 LI (\%) & $1.0(0.1-20.6)$ & $1.1(0.4-20.6)$ & $1.5 \pm 1.6$ \\
Mean \pm s.D. & $2.0 \pm 2.8$ & $2.7 \pm 4.0$ & 0.732 \\
\hline
\end{tabular}

MRI, magnetic resonance imaging; PRL, lactotroph adenoma; GH, somatotroph adenoma; ACTH, corticotroph adenoma; Gn, gonadotroph adenoma; Pluri, plurihormonal adenoma; Null, null cell adenoma.

${ }^{\mathrm{a} F i r s t}$ MRI.

significant difference (Table 1). However, post-operative tumor progression was slightly more frequently seen in functioning adenomas $(36.6 \% ; 15 / 41$ cases) than in non-functioning adenomas (32.8\%; 39/119 cases), while the MIB-1 LIs of progressive adenomas (median 1.1, mean 2.7) were slightly higher than those of non-progressive adenomas (median 0.9, mean 1.5). The MIB-1 LIs of functioning adenomas (median 1.0, mean 2.7) and non-functioning adenomas (median 0.9, mean 1.7) were also not significantly different $(P=0.138)$.

The interval between the first and second MRI studies was $30.5 \pm 27.1$ months (mean \pm s.D.; range 2-107.6 months). The tumor volume from the first MRI $\left(V_{1}\right)$ of these 54 cases was $2.9 \mathrm{ml}(0.001-44.4$; median and range). The median tumor volume of functioning adenomas $(1.1 \mathrm{ml})$ was smaller than that of nonfunctioning adenomas $(3.2 \mathrm{ml})$, and it showed borderline significance $(P=0.050$; Fig. 1a). The tumor volume measured from the second MRI $\left(V_{2}\right)$ was $4.4 \mathrm{ml}$ (0.1-71.3; median and range). The median tumor volume of functioning adenomas $(4.3 \mathrm{ml})$ was similar to that of non-functioning adenomas $(4.4 \mathrm{ml})$, and they showed no significant difference $(P=0.199$; Fig. $1 b)$.

The TVDT of the entire group ranged from 3.7 months ( 0.31 years) to 874.9 months (72.9 years) with a skew to the right (skewness $=4.09$ ). The mean TVDT was 78.7 months ( 6.56 years; s.D. $=148.3$ months), and the median TVDT was 34.6 months (2.88 years). The drastic difference in the mean and median was due to the skewness and the presence of a few extremely slow growing cases. The median TVDT of the functioning adenomas was 33.7 months and that of non-functioning adenomas was 35.4 months; they were not significantly different $(P=0.735$; Fig. $1 c)$.

The mean and median MIB-1 LI of the entire group were 2.7 and 1.1 respectively (range $0.4-20.6$; Table 2 ). Those of functioning tumors were 4.0 and 1.8 respectively (range 0.4-20.6), which were higher than those of non-functioning tumors (2.2 and 1.1; 0.4-19.4). However, they were not statistically significant $(P=0.374$; Fig. $1 \mathrm{~d})$.

The TVDT and MIB-1 LI of these 54 cases were not significantly different in different genders, adenoma type, and functional status of the tumor (Table 2). Although not reaching statistical significance, the median TVDT of younger patients $(\leq$ median, 48.5 years; 27.1 months) was shorter than that of older ( $>$ median) patients (42.7 months; $P=0.065$ ).

The MIB-1 LI was correlated with TVDT (Spearman's rank correlation coefficient, $\rho=-0.303, P=0.026$ ), but not linearly correlated (Pearson's product moment correlation coefficient, $r=-0.168, P=0.223)$. The optimal cut-off point of MIB-1 LI by ROC curve was $0.8 \%$. When LI $\geq 0.8$, the TVDT of $51.5 \%$ cases $(17 / 33)$ was $<2$ years, and when $\mathrm{LI}<0.8$, the TVDT of $90.5 \%$ cases $(19 / 21)$ was 2 or more years. The sensitivity and specificity of the prediction were $54.3 \%$ and $89.4 \%$ respectively. After log transformation, the MIB-1 LI was linearly correlated with $\log _{2}$ (TVDT) $(r=-0.363$, $P=0.007$; Table 3). This relationship showed borderline significance, while subgrouped into functioning and non-functioning adenomas. Using linear regression, the TVDT of pituitary adenoma could be estimated using the 

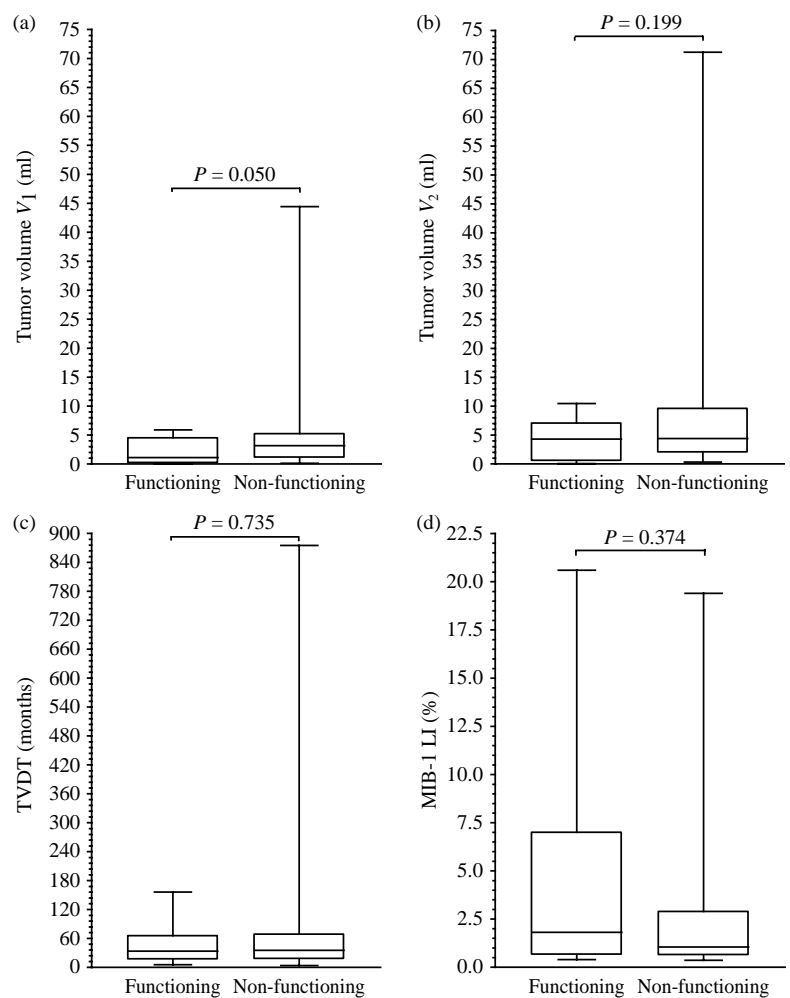

Figure 1 Box and whisker plots showing median, quartiles, and range of (a) tumor volume from the first MRI $\left(V_{1}\right)$, (b) tumor volume from the second MRI $\left(V_{2}\right)$, (c) tumor volume doubling time (TVDT), and (d) MIB-1 LI of the 54 post-operative tumor progression cases.

equation: TVDT $=2^{(5.569-0.157 \times \text { MIB- } 1}$ LI),$\quad P=0.007$. For example, when the MIB-1 LI of a pituitary adenoma is 3 , the estimated TVDT would be 34.2 months $(2.85$ years). The relationship between MIB-1 LI and $\log _{2}$ (TVDT) is shown as a scatter plot in Fig. 2.

\section{Discussion}

Prediction of post-operative tumor progression is of clinical importance since it could help in adjusting the timing of adjuvant radiotherapy. MIB-1 LI would be a candidate for this task. There were many studies in the literature using MIB-1 LI to study pituitary adenomas. However, there was no consensus as to its relation with growth velocity and tumor recurrence $(6,7,9-11,13$, 17-20, 23-25, 27). Therefore, we intended to evaluate whether MIB-1 LI actually related to growth rate of pituitary adenomas, which was measured by radiographic studies.

There were four studies in the literature correlating MIB-1 LI with growth velocity or TVDT calculated from serial image studies of pituitary adenomas $(7,19,20$, 27). Although three of them showed significant inverse correlation between LI and TVDT $(7,19,20)$, the TVDT of pituitary adenomas calculated in different series varied drastically, one being $930 \pm 180$ days (mean \pm s.D.; range 200-2550 days) (7) and the other being $1836 \pm 3445$ days (506-5378 days) (20). Part of the reasons for this discrepancy could be related to the limited number of study cases (14 cases (7), 23 cases (19), 38 cases (20), and 28 cases (27)), using a mixture of CT or MRI rather than a single form of image study for calculation of tumor size $(7,19)$, and only studying nonfunctioning adenomas $(7,19,20,27)$.

This study included all types of pituitary adenomas, both functioning and non-functioning, except for corticotroph adenoma and thyrotroph adenoma because of not fulfilling the selection criteria. We only used MRI rather than a mixture of CT and MRI to evaluate tumor volume because MRI has better resolution than $\mathrm{CT}$, and using a single method would eliminate the differences between two study methods and allow better comparison between two studies of the

Table 2 Tumor volume doubling time (TVDT) and MIB-1 LI of 54 cases of pituitary adenomas with post-operative tumor progression.

\begin{tabular}{|c|c|c|c|c|c|}
\hline & Total & TVDT (month) & $\boldsymbol{P}$ & MIB-1 LI (\%) & $P$ \\
\hline Case number & $54(100)$ & $34.6(3.7-874.9)$ & & $1.1(0.4-20.6)$ & \\
\hline Age (years) & $48.5(20-73)$ & & 0.065 & & 0.203 \\
\hline$\leq 48.5$ & $27(50.0)$ & $27.1(3.7-340.7)$ & & $2.0(0.4-19.4)$ & \\
\hline$>48.5$ & $27(50.0)$ & $42.7(4.9-874.9)$ & & $1.0(0.4-20.6)$ & \\
\hline Gender & & & 0.219 & & 0.890 \\
\hline Male & $28(51.9)$ & 42.6 (3.7-874.9) & & $1.2(0.5-8.4)$ & \\
\hline Female & $26(48.1)$ & $29.2(5.4-619.9)$ & & $1.1(0.4-20.6)$ & \\
\hline Adenoma type & & & 0.111 & & 0.460 \\
\hline PRL & $8(14.8)$ & $25.1(3.7-53.1)$ & & $2.0(0.4-6.6)$ & \\
\hline $\mathrm{GH}$ & $1(1.9)$ & 156.4 & & 2.5 & \\
\hline Gn & $23(42.6)$ & $56.3(4.9-874.9)$ & & $1.0(0.4-6.5)$ & \\
\hline Pluri & 17 (31.5) & $36.0(5.4-619.9)$ & & $1.3(0.6-20.6)$ & \\
\hline Null & $5(9.3)$ & $26.8(6.0-50.0)$ & & $0.5(0.4-19.4)$ & \\
\hline Functional status & & & 0.735 & & 0.374 \\
\hline Functioning & $15(27.8)$ & $33.7(5.4-156.4)$ & & $1.8(0.4-20.6)$ & \\
\hline Non-functioning & 39 (72.2) & $35.4(3.7-874.9)$ & & $1.1(0.4-19.4)$ & \\
\hline
\end{tabular}

Data represented as $n(\%)$ or median (range).

LI, labeling index (\%); PRL, lactotroph adenoma; GH, somatotroph adenoma; Gn, gonadotroph adenoma; Pluri, plurihormonal adenoma; Null, null cell adenoma. 
Table 3 Correlation between MIB-1 labeling index and $\log _{2}$ (tumor volume doubling time) of pituitary adenoma.

\begin{tabular}{lccc}
\hline & & $\begin{array}{c}\text { Pearson's } \\
\text { correlation } \\
\text { coefficient }\end{array}$ & $\boldsymbol{P}$ \\
\hline Pituitary adenoma & $54(100)$ & -0.363 & 0.007 \\
Functioning & $15(27.8)$ & -0.513 & 0.050 \\
Non-functioning & $39(72.2)$ & -0.313 & 0.053 \\
\hline
\end{tabular}

same case and between different cases. Although we intended to include more cases in this study, there were only 54 cases $(9.5 \%)$ out of a total of 596 pituitary adenoma cases that had undergone surgery and fulfilled the selection criteria during the 11-year case collection period. It is, therefore, understandable why there were only a few reports concerning this subject and studying relatively small number of cases in the literature $(7,19,20)$.

It was noted in our observation that post-operative tumor progression only occurred in around one-third of the cases $(33.8 \%)$. Of the 160 cases that had two postoperative MRI studies and did not receive other adjuvant therapies, $106(66.3 \%)$ cases had a residual tumor size that was either unchanged or shrunk further, and the cause of the latter finding could be related to vascular damage due to surgery. All the parameters analyzed between progressive and nonprogressive groups, e.g. age, gender, adenoma types, functional status, initial tumor volume, and MIB-1 LIs, were not significantly different.

Excluding one case that exceeded the mean +4 S.D., the mean TVDT of the 53 cases within the mean +2 s.D. in our series was $63.7 \pm 100$ months (1910 \pm 3000 days; 5.31 years), which was very close to

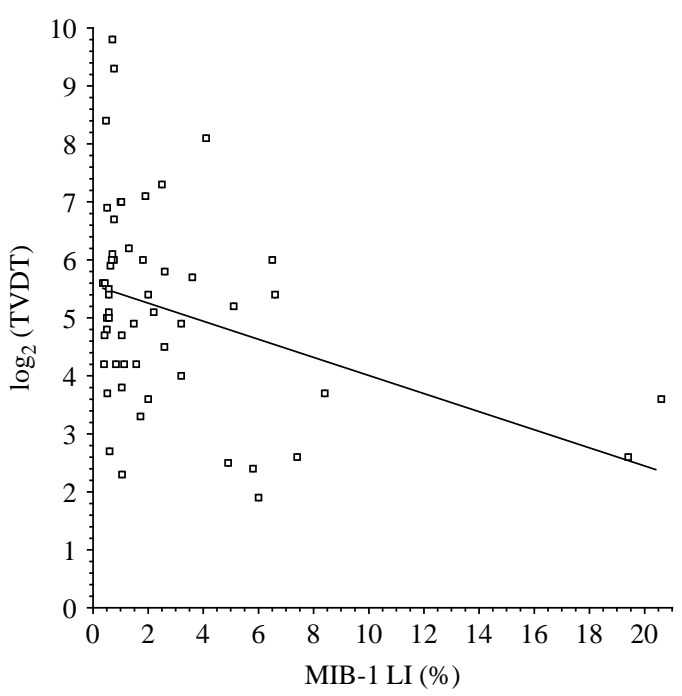

Figure 2 The distribution of MIB-1 LI and $\log _{2}$ (TVDT) of 54 postoperative tumor progression pituitary adenomas. that reported by Tanaka et al. being $1836 \pm 3445$ days (20). As speculated by Tanaka et al., the much shorter mean TVDT, $930 \pm 180$ days, reported by Ekramullah et al. (7) could be due to younger age of their patients ( $48 \pm 3.2$ years; $57.1 \pm 15.7$ years in Tanaka's series), since the TVDT of the younger patients $(<61$ years) of Tanaka's series was 1106 days (20). However, the age of our study group, which was $49.4 \pm 15.8$ (median 48.5) years, was similar to that of Ekramullah's series.

From the data in the literature and in our study, it was noted that except for a few cases that had high MIB-1 LI, most pituitary adenomas had low proliferation potential $(7,9,12,13,15-25)$. The MIB-1 LI of pituitary adenomas reported in different studies varied presumably due to differences in the staining and counting methodologies $(7,9,12,13,15-25)$. The reported mean ranged from 0.84 to $2.72(7,9,12$, $15-17,19,20,23-25)$, the median ranged from 1.50 to $1.80(12,14,22,23)$, and the range was from 0 to $23.6(7,9,13,15,18-23,25)$. The mean, median, and range of MIB-1 LI in our series were 2.7, 1.1, and 0.4-20.6 respectively, and were similar to those reported. In our series, the LI of functioning tumors was higher than that of non-functioning tumors; however, the difference was not statistically significant.

There has been no consensus concerning the correlation between MIB-1 LI and pituitary adenoma progression in the literature. While some studies showed MIB-1 LI to be an independent predictor of progression $(19,24,25)$, others did not $(11,13,18$, 23). Although not reaching statistical significance, our study found that the MIB-1 LI of progressive adenomas (median 1.1, mean 2.7) was slightly higher than that of non-progressive adenomas (median 0.9, mean 1.5).

Our study demonstrated a correlation between MIB-1 LI and $\log _{2}$ (TVDT) in pituitary adenomas, and an equation was formulated to estimate TVDT with the use of MIB-1 LI. However, it was complicated and difficult to use in clinical practice.

Because of the wide range of TVDTs and MIB-1 LIs reported in the literature, it is unlikely to form a universally accepted MIB-1 LI cut-off value to distinguish progressive and non-progressive pituitary adenomas. Nevertheless, we proposed using MIB-1 LI of 0.8 as a threshold to alert for possible post-operative tumor progression, since the optimal cut-off point of MIB- 1 LI by ROC curve was $0.8 \%$, and the mean MIB- 1 LIs reported in the literature and in this study were $>0.8$ (ranged from 0.84 to 2.72$)(7,9,12,15-17$, 19-21, 23-25). For our studied pituitary adenomas with MIB-1 LI $\geq 0.8$, the TVDT of $51.5 \%$ cases was $<2$ years, and for tumors with MIB- 1 LI $<0.8$, the TVDT of $90.5 \%$ cases was 2 or more years. While the sensitivity of the prediction was low $(54.3 \%)$, the specificity was acceptable (89.4\%).

Besides MIB-1 LI, the functioning status of pituitary adenoma was also correlated with post-operative tumor progression. A previous study has shown that 
functioning adenomas were less frequently progressed in size than non-functioning adenomas (25 vs $58 \%$, $P=0.001$ ) during post-operative follow-up (25). Our observation did not reveal a significant difference and showed that post-operative tumor progression in functioning adenomas and non-functioning adenomas were 36.6 and $32.8 \%$ respectively $(P=0.656)$. The MIB-1 LI of our functioning adenomas (median 1.8) was slightly higher than that of non-functioning adenomas (1.1), although it was not statistically significant. It has also been reported that the MIB-1 LI of functioning adenomas was significantly higher than that of non-functioning adenomas (21). It is also possible that the functioning adenomas in our study might not be representative for all functioning adenomas, since tumors that were larger and invasive usually had received adjuvant radiotherapy and were excluded from this study.

In conclusion, our data showed that most pituitary adenomas have low proliferative potential, and their MIB-1 LIs were significantly correlated with the MRI detected TVDT. Post-operative tumor progression occurred in only one-third of cases, while the rest were either stable or underwent further shrinkage. Although it was difficult to set an absolute threshold to predict post-operative progression, MIB-1 LI of $<0.8$ indicated a slower growing tumor.

\section{Declaration of interest}

The authors declare that there is no conflict of interest that could be perceived as prejudicing the impartiality of the research reported.

\section{Funding}

This work was supported by the Taipei Veterans General Hospital (grant numbers V96C1-001, V97C1-099) and the National Science Council (grant number NSC 96-2320-B-075-004-MY2).

\section{Author contribution statement}

C-Y H read the immunohistochemistry, did the statistics, and wrote the manuscript; $\mathrm{W}-\mathrm{Y} \mathrm{G}$ and C-P C reviewed the MRI; D M-T H designed the research, read the immunohistochemistry, and revised the manuscript.

\section{Acknowledgements}

The authors thank Ms Windy Wen-Chi Huang for her technical assistance.

\section{References}

1 Hoshi M, Hayashi T, Kagami H, Murase I \& Nakatsukasa M. Late bilateral temporal lobe necrosis after conventional radiotherapy. Neurologia Medico-Chirurgica 200343 213-216.

2 Piatt JH Jr, Blue JM, Schold SC Jr \& Burger PC. Glioblastoma multiforme after radiotherapy for acromegaly. Neurosurgery 1983 $1385-89$.
3 Bhansali A, Banerjee AK, Chanda A, Singh P, Sharma SC, Mathuriya SN \& Dash RJ. Radiation-induced brain disorders in patients with pituitary tumours. Australasian Radiology $2004 \mathbf{4 8}$ 339-346.

4 Gerdes J, Becker MH, Key G \& Cattoretti G. Immunohistological detection of tumour growth fraction (Ki-67 antigen) in formalinfixed and routinely processed tissues. Journal of Pathology 1992 168 85-86.

5 Cattoretti G, Becker MH, Key G, Duchrow M, Schluter C, Galle J \& Gerdes J. Monoclonal antibodies against recombinant parts of the Ki-67 antigen (MIB 1 and MIB 3) detect proliferating cells in microwave-processed formalin-fixed paraffin sections. Journal of Pathology $1992168357-363$.

6 Kawamoto H, Uozumi T, Kawamoto K, Arita K, Yano T \& Hirohata T. Analysis of the growth rate and cavernous sinus invasion of pituitary adenomas. Acta Neurochirurgica 1995136 37-43.

7 Ekramullah SM, Saitoh Y, Arita N, Ohnishi T \& Hayakawa T. The correlation of Ki-67 staining indices with tumour doubling times in regrowing non-functioning pituitary adenomas. Acta Neurochirurgica 1996138 1449-1455.

8 Thapar K, Kovacs K, Scheithauer BW, Stefaneanu L, Horvath E, Pernicone PJ, Murray D \& Laws ER Jr. Proliferative activity and invasiveness among pituitary adenomas and carcinomas: an analysis using the MIB-1 antibody. Neurosurgery $1996 \mathbf{3 8}$ 99-106. discussion 106-107.

9 Abe T, Sanno N, Osamura YR \& Matsumoto K. Proliferative potential in pituitary adenomas: measurement by monoclonal antibody MIB-1. Acta Neurochirurgica 1997139 613-618.

10 Mizoue T, Kawamoto H, Arita K, Kurisu K, Tominaga A \& Uozumi T. MIB1 immunopositivity is associated with rapid regrowth of pituitary adenomas. Acta Neurochirurgica 1997139 426-431. discussion 431-432.

11 Yonezawa K, Tamaki N \& Kokunai T. Clinical features and growth fractions of pituitary adenomas. Surgical Neurology 1997 $\mathbf{4 8} 494-500$.

12 Mastronardi L, Guiducci A, Spera C, Puzzilli F, Liberati F \& Maira G. Ki-67 labelling index and invasiveness among anterior pituitary adenomas: analysis of 103 cases using the MIB-1 monoclonal antibody. Journal of Clinical Pathology 199952 $107-111$.

13 Losa M, Franzin A, Mangili F, Terreni MR, Barzaghi R, Veglia F, Mortini P \& Giovanelli M. Proliferation index of nonfunctioning pituitary adenomas: correlations with clinical characteristics and long-term follow-up results. Neurosurgery $2000 \quad \mathbf{4 7}$ 1313-1318. discussion 1318-1319.

14 Mastronardi L, Guiducci A, Spera C, Puzzilli F, Liberati F, Ruggeri A \& Peciarolo A. Adrenocorticotropic hormone secreting pituitary adenomas: analysis of growth fraction using the MIB-1 antibody. Tumori 200086 229-232.

15 Lath R, Chacko G \& Chandy MJ. Determination of Ki-67 labeling index in pituitary adenomas using MIB-1 monoclonal antibody. Neurology India 200149 144-147.

16 Mastronardi L, Guiducci A \& Puzzilli F. Lack of correlation between Ki-67 labelling index and tumor size of anterior pituitary adenomas. BMC Cancer 2001112.

17 Jaffrain-Rea ML, Di Stefano D, Minniti G, Esposito V, Bultrini A, Ferretti E, Santoro A, Faticanti Scucchi L, Gulino A \& Cantore G. A critical reappraisal of MIB-1 labelling index significance in a large series of pituitary tumours: secreting versus non-secreting adenomas. Endocrine-Related Cancer 20029 103-113.

18 Hentschel SJ, McCutcheon E, Moore W \& Durity FA. P53 and MIB-1 immunohistochemistry as predictors of the clinical behavior of nonfunctioning pituitary adenomas. Canadian Journal of Neurological Sciences $200330215-219$.

19 Honegger J, Prettin C, Feuerhake F, Petrick M, Schulte-Monting J \& Reincke M. Expression of Ki-67 antigen in nonfunctioning pituitary adenomas: correlation with growth velocity and invasiveness. Journal of Neurosurgery 200399 674-679. 
20 Tanaka Y, Hongo K, Tada T, Sakai K, Kakizawa Y \& Kobayashi S. Growth pattern and rate in residual nonfunctioning pituitary adenomas: correlations among tumor volume doubling time, patient age, and MIB-1 index. Journal of Neurosurgery $2003 \mathbf{9 8}$ 359-365.

21 Wolfsberger S, Kitz K, Wunderer J, Czech T, Boecher-Schwarz HG, Hainfellner JA \& Knosp E. Multiregional sampling reveals a homogenous distribution of Ki- 67 proliferation rate in pituitary adenomas. Acta Neurochirurgica 2004146 1323-1327. discussion 1327-1328.

22 Wolfsberger S, Wunderer J, Zachenhofer I, Czech T, BocherSchwarz HG, Hainfellner J \& Knosp E. Expression of cell proliferation markers in pituitary adenomas - correlation and clinical relevance of MIB-1 and anti-topoisomerase-IIalpha. Acta Neurochirurgica 2004146 831-839.

23 Dubois S, Guyetant S, Menei P, Rodien P, Illouz F, Vielle B \& Rohmer V. Relevance of Ki-67 and prognostic factors for recurrence/progression of gonadotropic adenomas after first surgery. European Journal of Endocrinology 2007157 141-147.

24 Fusco A, Zatelli MC, Bianchi A, Cimino V, Tilaro L, Veltri F, Angelini F, Lauriola L, Vellone V, Doglietto F, Ambrosio MR, Maira G, Giustina A, degli Uberti EC, Pontecorvi A \& De Marinis L.
Prognostic significance of the Ki-67 labeling index in growth hormone-secreting pituitary adenomas. Journal of Clinical Endocrinology and Metabolism 200893 2746-2750.

25 Gejman R, Swearingen B \& Hedley-Whyte ET. Role of Ki-67 proliferation index and p53 expression in predicting progression of pituitary adenomas. Human Pathology 200839 758-766.

26 Schreiber S, Saeger W \& Lüdecke DK. Proliferation markers in different types of clinically non-secreting pituitary adenomas. Pituitary 19991 213-220.

27 Saeger W, Lüdecke B \& Lüdecke DK. Clinical tumor growth and comparison with proliferation markers in non-functioning (inactive) pituitary adenomas. Experimental and Clinical Endocrinology and Diabetes 2008116 80-85.

28 Nakaguchi H, Fujimaki T, Matsuno A, Matsuura R, Asai A, Suzuki I, Sasaki T \& Kirino T. Postoperative residual tumor growth of meningioma can be predicted by MIB-1 immunohistochemistry. Cancer 199985 2249-2254.

Received 19 February 2010

Accepted 15 March 2010 